\title{
PERFORMANCE ANALYSIS OF A ZIGBEE BEACON ENABLED CLUSTER TREE NETWORK
}

\author{
Saad A. Khan ${ }^{1}$ and Fahad A. Khan ${ }^{2}$ \\ ${ }^{1}$ Al-Khwarizmi Institute of Computer Science University of Engineering \& Technology, Lahore, Pakistan. \\ ${ }^{2}$ Department of Electrical Engineering, University of Engineering \& Technology, Lahore, Pakistan. \\ saad.ahmad@uet.edu.pk, fahad.ahmad@uet.edu.pk
}

\begin{abstract}
Zigbee provides a realistic and feasible solution for the implementation of 'low data rate', 'low cost' and 'minimum energy consumption' networks. These attractive features are compelling the industry to adopt Zigbee and deploy and integrate it in a whole slew of consumer market applications. Energy consumption always plays a critical role in modern system design and coerces developers to propose and exploit various routing techniques to minimize power losses. The IEEE 802.15.4 standard has been made use of for the MAC and physical layers of the Zigbee protocol stack. In our work we have identified that a clustered network approach brings about minimum energy consumption in such networks; this is owes to the fact of load distribution among data aggregating heads. We provide a beacon enabled performance analysis of such network. The impact of Zigbee's deployment has been felt in e.g. the health care industry, patient monitoring, home are networks, remote surveillance and management and consumer wireless devices such as cell phones, PDA's, etc. We expect our findings to aid in developers in engineering systems in the aforementioned applications; in such a manner that Zigbee's integration would result in networks that consume minimal energy, thereby, conserving energy and ensuring maximal longevity of the network and its devices.
\end{abstract}

Index Terms-Zigbee; IEEE 802.15.4; Performance Analysis in IEEE 802.15.4; Multi hop network; Home Area Network

\section{INTRODUCTION}

WIRELESS sensor network (WSN) is an emerging technology comprising of complex distributed network of nodes that have sensing, data processing and storage capabilities. Nodes in sensor networks have trammeled amount of energy for their operations. WSN are usually composed of numerous nodes that sense surroundings, transmitting information wireless to other nodes for sharing collaborative tasks. Recent advancement in this field has provided with standards such as IEEE 802.15.4 and Zigbee, which provides a platform for many commercial applications like environmental, health, home control etc. One of the areas where wireless sensor network and consumer electronics blend together is home automation and home area networking. One major objective is to develop an integrated and user-friendly environment with least power consumption and long battery life [1].

Recent research in clustered wireless sensor networks shows the importance of establishing fusing head formations to preserve energy. Such formations help in increasing the network life-time. In clustered sensor network the sensed data is not directly sent to the coordinator (sink node), but to designated cluster head which collect the data for nodes in cluster to route it to sink node via shortest paths of other heads. Routing in Zigbee networks is quite similar to ones used in Mobile Adhoc networks (MANET's), in which sustaining end-to-end efficient routing paths in challenging due to many factor including orphaning of nodes due to node failures, mobility issues etc [2].

Most of the industrial applications have parallels in home automation. For example, a home HVAC that is equipped with temperature and light sensors can keep the inside of room moderated according to outside sunny side and temperature. One important use of Zigbee in such networks is due to its large support of network. With such implementation and location-aware capabilities a diverse collection of consumer activities can be entertained. Location can provide contextspecific information in activities like shopping, tourism [3].

For management of routing paths in home area networking, quite complex computations are required. Use of clustered topology eliminates redundancy in such a way that only accumulated data is sent to the sink. While using Zigbee, the unique active, sleep and wake-up modes of deployed nodes ultimately result in power saving. Possible network topologies for Zigbee sensor networks include star, cluster-tree and mesh topology respectively. Zigbee networks are usually data centric based on the concept of attributes and clusters. Hierarchical routing and clustering has due advantages of scalability, self organization, security, better data aggregation and fault tolerance. Although hierarchical clustering is well known for large sized and full-load networks yet we are now using it for HAN`s. In hierarchical architecture, there is less computation burden, dynamic organization, localization and redundancy exploitation [4].

In this paper we provide the mathematical analysis of a beacon enabled clustered multi-hop network. Performance analysis of coordinator and end-devices has been done in a beacon enabled configurable. Further the life time of nodes has been calculated using real analysis of Zigbee nodes. The nodes used for this purpose are IEEE 802.15.4 complaint Chipcon CC2431.

The organization of paper is as follows: Section II is the related work that had been done on the performance analysis of IEEE 802.15.4. Section III gives a brief introduction to Zigbee with respect to data transmission. Section IV comprises performance analysis of the Zigbee cluster tree network. We conclude the paper in section $\mathrm{V}$. 


\section{RELATED WORK}

A large scale star network mathematical analysis has been presented by Bougard [6]. The special contribution made by this paper is the BER calculation, where operational analysis has been focused to packet size of data and path loss. In [20], authors have proposed model for the slotted Carrier Sense Multiple Access with Collision Avoidance (CSMA/CA) access scheme of the IEEE 802.15.4 standard for the unacknowledged transmission mode. In [7], it has been found that using low-duty-cycle operations, energy can be saved. Performance Analysis of IEEE 802.15.4 in a cluster tree network has been presented in [11]. Beacon enabled networks are prone to beacon collisions, which definitely leads to synchronization failures. Such beacon collisions can be minimized by using long beacon intervals. Moreover, using more frequency channels is an option for reduction in collisions. Yet using such options always culminates in an increased energy consumption of network. A deep performance probabilistic analysis and modeling of IEEE 802.15.4 for medical area networking has been done by Timmons and Scanlon [8]. Basic formulae and calculations for average back off, frame size, node lifetime and reception and transmission times have been done thoroughly.

In [9], a detailed performance analysis of IEEE 802.15.4 has been done for a cluster-tree based topology (pure mesh networking). Device power consumption, node operations, calculation of back off time, coordinator's energy efficiency \& good put have been modeled \& simulated using WISENES.

In [12] J. Misic and her group have analyzed the IEEE Standard 802.15.4 in the beacon enabled mode using the theory of discrete time Markov chains and $\mathrm{M} / \mathrm{G} / 1 / \mathrm{K}$ queues. The paper includes the impact of different parameters such as packet arrival rate, number of stations, station's buffer size, packet size, and inactive period between the beacons. Several important performance parameters such as probability of access, probability that medium is idle, queue length distribution in the device, and probability distribution of the packet service time is also discussed in the paper. Lately, her group has done an extensive work related to analysis of cluster interconnection schemes in 802.15 .4 beacon enabled networks in [17-19].

\section{ZigbeE BeAcon EnABled MOde OvervieW}

Zigbee protocol stack is divided into multiple layers as shown in figure 1. The top most is the application layer which is responsible for a node's relationship in the network; it also supervises and initiates the network. ZDO (Zigbee Device Object) performs the overall node management. There are 255 endpoints defined in the layer, ' 0 ' end-point being specified for ZDO, which can be called upon in order to discover other Zigbee nodes, to see profiles, to define security and network settings. Security functions like encryption, authentication, key distribution and other security related functionality is handled by the SSP.

The NWK layer is responsible for providing the multi hop and routing facilities. NWK can be initiated and other functions like route discovery, maintenance and message relaying functions are done in this layer.

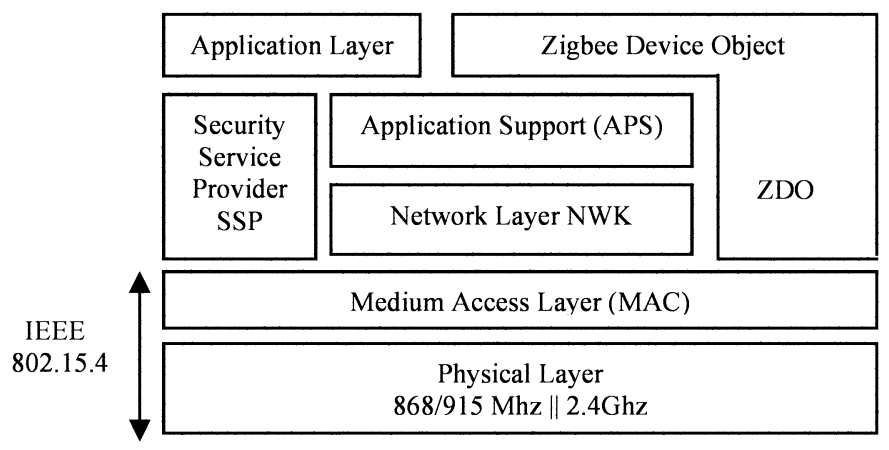

Fig. 1. Zigbee Protocol Stack \& IEEE 802.15.4

There is an additional layer between the network and application layer i.e. the APS layer. It connects the NWK, $\mathrm{SSP}$, endpoints and is responsible to route messages to different sub layers.

IEEE 802.15.4 has defined the MAC and PHY layers are shown in the figure. MAC is responsible network association, channel access mechanism, acknowledged frame delivery etc. There are two DSSS (Direct Spread Spectrum Sequence) physical layer supported by IEEE 802.15.4 in ISM (Industrial, Scientific and Medical) frequency bands. Low-band operates in the $868 \mathrm{Mhz}$ or $915 \mathrm{Mhz}$ frequency band. The raw data rate of 20 or $40 \mathrm{Kbps}$ is supported by it. A high data rate of 250 $\mathrm{Kbps}$ is supported in high frequency band of $2.4 \mathrm{Ghz}$ [5].

Since the main goal of a Zigbee network is data transmission under the constraint of maximum power saving, a beacon frame structure can be employed. In the beaconenabled mode, all communications are performed in a superframe structure. A superframe is bounded by periodically transmitted beacon frames, which allow nodes to synchronize to the network. An active part of a superframe is divided into 16 contiguous time slots that form three parts: the beacon, Contention Access Period (CAP) and Contention-Free Period (CFP). At the end of the superframe is an inactive period, when nodes may enter to a power saving mode. The Beacon Interval $\left(I_{B}\right)$ and the active Superframe Duration $(S D)$ are adjustable by Beacon Order $(B O)$ and Superframe Order $(S O)$ parameters as

$B I=$ aBaseSuperframeDuration $\times 2^{B O}$

$S D=$ aBaseSuperframeDuration $\times 2^{S O}$

(1)

In addition to the beacon, CAP is a mandatory part of a superframe. Coordinators are required to listen to the channel the whole CAP to detect and receive any data from their child nodes. On the other hand, the child nodes may only transmit data and receive an optional acknowledgement (ACK) when needed, which increases their energy efficiency. In the contention access period (CAP), every node can transmit according to the CSMA/CA MAC protocol [14], with the use of a proper backoff algorithm [15], as required by the IEEE 802.15.4 standard. 
CFP is an optional feature of IEEE 802.15.4 MAC, in which a channel access is performed in allocated time slots. In order to allow safe data transmission, a guaranteed time slot (GTS) may be reserved to nodes which require it [16]. A node may reserve bandwidth for delay critical applications by requesting GTS from a PAN Coordinator. The GTS allocations are signaled in beacon frames. In star networks, a device may obtain better Quality of Service (QoS) by the use of GTS, since contention and collisions are avoided. Yet, the applicability of GTS in peer-to-peer or cluster-tree networks is poor, since GTS may be used only between the PAN Coordinator and its one-hop neighbors. Moreover, intercoordinator collisions degrade QoS in GTS, since no collision avoidance mechanism is used in CFP.

\section{Performance AnAlysis}

To analyze the performance of devices in cluster tree network we have done analysis for end-devices and network coordinator. The models being used are based on analysis done in cluster tree network, presented in [9]. To obtain real deployment results, Chipcon's CC2431 module has been used for performance analysis. This module contains a SOC solution of Zigbee networks. It is an extension to their previous device CC2340 with additional unit of localization [10].

\section{Configuration of cluster tree network:}

To obtain the lowest power consumption, a cluster-tree type beacon-enabled network topology is selected. In addition, CFP option is not used and all data exchanges are performed during CAP. Thus, the CAP length $\left(t_{C A P}\right)$ is approximated to be equal to the superframe length.

To analyze the cluster tree network, we consider a network which has three child coordinators and within each cluster are twelve devices. The network depth has been taken as four, which results in a total of $1500+$ nodes. Data is transmitted from each node to the cluster head which routes the data to the network coordinator. This activity corresponds to the uplink data. The interval between the transmission frames is normalized to the beacon interval of the superframe.

Due to the effect to interlacing as defined in Zigbee specifications, we have ignored the beacon collisions. In Zigbee specification time is divided into periodic slots of equal length to the superframe length. At the startup of network the coordinator performs passive scan and performs operation of searching for neighbors and spare slots for periodic transmissions. A randomly selected slot is used from the acquired free slots to avoid the collisions with siblings.

The two essential network parameters that are required for the determination of duty cycle are the beacon order $B O$ and the super frame order $S O$. In a beacon-enabled network, TABLE I

MEASURED POWER CONSUMPTION @ 2.7 V

\begin{tabular}{lcc}
\hline \hline Symbol & Current & Power Consumption \\
\hline $\mathrm{P}_{\mathrm{TX}}$ & $26 \mathrm{~mA}$ & $70 \mathrm{~mW}$ \\
$\mathrm{P}_{\mathrm{RX}}$ & $29 \mathrm{~mA}$ & $78.3 \mathrm{~mW}$ \\
$\mathrm{P}_{\mathrm{CCA}}$ & $30.7 \mathrm{~mA}$ & $83 \mathrm{~mW}$ \\
$\mathrm{P}_{\mathrm{I}}$ & $1.4 \mathrm{~mA}$ & $3.79 \mathrm{~mW}$ \\
$\mathrm{P}_{\mathrm{MCU}}$ & $0.8 \mathrm{~mA}$ & $2.25 \mathrm{~mW}$ \\
$\mathrm{P}_{\mathrm{S}}$ & $0.06 \mathrm{uA}$ & $1.62 \mu \mathrm{W}$ \\
& & \\
\hline \hline
\end{tabular}

device receives beacons and exchanges data with a coordinator. The rest of time, device is in the sleep mode. Table I gives the measured power consumption of CC2431

\section{Performance Analysis of RFD:}

Two types of configuration can be supported in a beacon enabled mode. One is query based data uplink. In this mode the sensor node wakes up during the beacon, sends back the data to cluster head and goes to sleep again. If the node would awake on every beacon interval, more energy will be drained which is not desired. It is due to the fact that reduced function device has more stringent power consumption as compared to fully functional device (i.e. coordinator).

The other configuration mode is periodic transmissions of data which can be accomplished by using a software operated timer of 8051 microcontroller built within the chip. Operating

TABLE II

ASSUMPTIONS

\begin{tabular}{lcc}
\hline \hline Symbol & & Value \\
\hline$A$ & Items in long frame & 8 \\
$R$ & Radio Data Rate & $250 \mathrm{kbps}$ \\
$t_{l}$ & Synchronization in & $100 \mu \mathrm{s}$ \\
& accuracy & $15.36-61.44 \mathrm{~ms}$ \\
$\mathrm{t}_{\mathrm{CAP}}$ & Contention access period & $960 \mu \mathrm{s}$ \\
$\mathrm{SD}$ & aBasesuperframeDuration & $20 \mathrm{ppm}$ \\
$\mathrm{Etx}, \mathrm{r}$ & Crystal tolerances &
\end{tabular}

$\mathrm{h}=$ hours, $\mathrm{s}=$ seconds;

the microcontroller in 8-bit auto-reload mode helps in obtaining required time intervals. When the reduced function device is awake, a MLME-SYNC.request is generated with the TrackBeacon parameter as off. This will trigger a onetime SYNC request due to which the sensor can receive the beacon from fully functional device, then transmit the payload data and go back into sleep mode. The data assumed for our analysis is of fifty bytes.

The duty cycle of device [9] is calculated in terms of various beacon receptions. Direct and indirect data transmissions and receptions have also been catered in calculations

$D C_{\text {device }}=\frac{t_{B R}}{B I}+\frac{\left(t_{S T}+t_{A R}\right) R e t}{U I \times B I}+\frac{\left(t_{S T}+t_{A R}+t_{R I}+t_{A T}\right) R e t}{D I \times B I}+$
$t_{N S} / \stackrel{\underline{ } S I}{ }$

(3)

where, $t_{B R}$ is Beacon reception time and is given by

$t_{B R}=t_{S I}+t_{I R}+\left(\epsilon_{T X}+\epsilon_{R X}\right) B I+t_{1}+\frac{L_{B}}{R}$

$t_{S T}$ is the transmission time for short frames. Usual applications in home network invoke short data frame length packets.

$t_{S T}=t_{S I}+t_{B O T}+t_{I T}+\frac{L_{S}}{R}$

The acknowledge $A C K$ reception time $t_{A R}$ and indirect data $t_{R I}$ transmission time are: 
$t_{A R}=t_{T R}+\frac{t_{A W}}{2}+\frac{L_{A}}{R}+t_{I F S}$

$t_{R I}=t_{1}+\frac{\left(t_{R E S}+t_{B O T}\right)}{2}+\frac{L_{S}}{R}$

ACK transmission time is obtained by adding up receive to transmit time, wait duration and ACK frame length.

$t_{A T}=t_{R T}+\frac{t_{A W}}{2}+\frac{L_{A}}{R}$

Network scanning time $t_{N S}$ either accounts for a new node which has to join the network or the node which has lost its contact.

$t_{N S}=t_{I R}+$ aBasesuperframeDuratio $\times\left(2^{B O}+\right.$ 1)

$P_{\text {device }}=\frac{E_{B R}}{B I}+\frac{\left(E_{S T}+E_{A R}+E_{R I}+E_{A T}\right) R e t}{D I \times B I}+\frac{\left(E_{S T}+E_{A R}\right) R e t}{U I \times B I}+\frac{E_{N S}}{N S I}+$ $\left(1-D C_{\text {devic 浜 }}\right) P_{s}$

(10)

where,$E_{B R}$ Beacon reception energy, $P_{\text {device }}$ is the power consumption of device

$E_{B R}=\left(t_{B R}-t_{S I}\right) P_{R X}+t_{S I} \times P_{1}$

Transmission energy $E_{S T}$ for short frames is

$E_{S T}=t_{S I} \times P_{1}+E_{B O T}+\left(t_{I T}+L_{S} / R\right) P_{T X}$

The energy required that is required for indirect communication of short frames is

$E_{R I}=t_{R I} \times P_{R X}$

Energies for ACK reception and transmission are

$E_{A R}=\left(t_{A R}-t_{I F S}\right) P_{R \text { 浜 }}+\left(t_{I F S} \times P_{1}\right)$

(14)

$E_{A T}=\left(t_{R T}+\frac{L_{A}}{R}\right) P_{T X}+\left(t_{A W} / 2\right) P_{1}$

respectively and network scanning energy is formulated as

$E_{N S}=t_{N S} \times P_{R X}$

Total ON time of the node $=30.26 \mathrm{~ms}$

Total active mode power consumption $=492.64$ (mA.ms)

Total low-power mode time of node

$=1 \mathrm{~min}-30.26 \mathrm{~ms}=59969.74 \mathrm{~ms}$

Total current consumption in low power mode

$=500 \mu \mathrm{A} \times 59969.74 \mathrm{~ms}=29.98487 \mathrm{~mA} . \mathrm{ms}$

Total active power consumption + inactive power consumption $=522.62 \mathrm{~mA} . \mathrm{ms}$

$=1.45 \times 10^{-4} \mathrm{mAh} / \mathrm{min}=0.00871 \mathrm{~mA}$

Assuming battery of 3000-mAh true capacity we have, $3000 / 0.00871 \mathrm{~mA}=344431$ hours approx $=39$ years approx which exceeds the shell life of AA battery.

Assuming coin-cell battery with $50 \mathrm{mAh}$ true capacity $50 \mathrm{mAh} / 0.00871 \mathrm{~mA}=5740$ hours approx $=239$ days
Figure 2, shows that less energy is required in searching the network as the beacon interval increases. Beacon order $B O$ which basically determines beacon interval $B I$ has atypical range of 6-10.We have calculated power consumption for $\mathrm{BO}=6$ and $\mathrm{SO}=0$. For small multi hop networking applications like home area networking, this value suffices as we have short frame lengths in such applications. It was observed during calculations that power consumption increases in case of higher BO. The dotted line is figure below gives the logarithmic trend for power consumption.

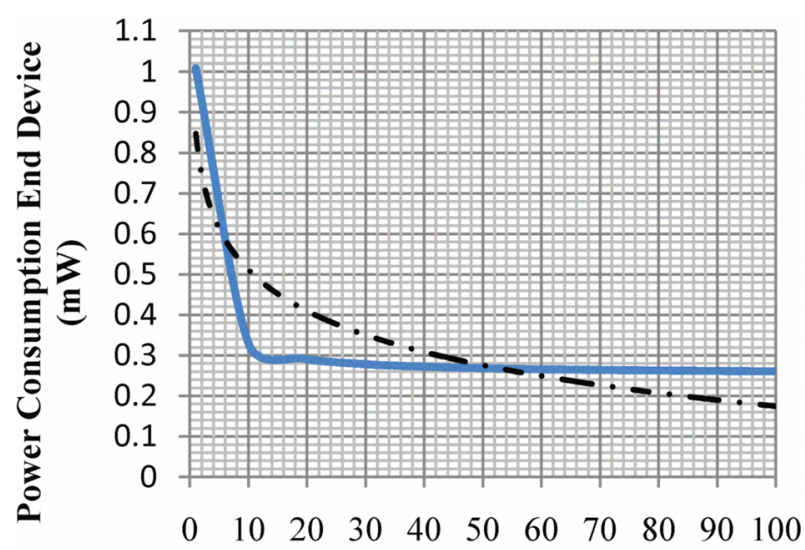

Beacon Interval (ms)

Fig. 2. RFD power consumption as a function of beacon interval

\begin{tabular}{|l|c|c|c|}
\hline \multicolumn{1}{|c|}{ Description } & Current & Duration & $\begin{array}{c}\text { Consumption } \\
\text { (mA.ms) }\end{array}$ \\
\hline $\begin{array}{l}\text { Startup Sequence. } \\
\text { MCU in active } \\
\text { mode running on } \\
16 \text { Mhz }\end{array}$ & $5 \mathrm{~mA}$ & $0.49 \mathrm{~ms}$ & 2.45 \\
\hline $\begin{array}{l}\text { MCU ON, running } \\
\text { on 32Mhz clock }\end{array}$ & $12 \mathrm{~mA}$ & $10.5 \mathrm{~ms}$ & 126.00 \\
\hline Radio on RX mode & $26 \mathrm{~mA}$ & $4.2 \mathrm{~ms}$ & 109.20 \\
\hline $\begin{array}{l}\text { Packet processing } \\
\text { MCU on }\end{array}$ & $12 \mathrm{~mA}$ & $2 \mathrm{~ms}$ & 24.00 \\
\hline Radio on RX mode & $27 \mathrm{~mA}$ & $1.95 \mathrm{~ms}$ & 52.65 \\
\hline Radio on, TX mode & $29 \mathrm{~mA}$ & $2 \mathrm{~ms}$ & 58.00 \\
\hline $\begin{array}{l}\text { Radio on, RX mode } \\
\text { for listening } \\
\text { acknowledgement }\end{array}$ & $26 \mathrm{~mA}$ & $1 \mathrm{~ms}$ & 26.00 \\
\hline MCU ON & $12 \mathrm{~mA}$ & $7.5 \mathrm{~ms}$ & 90.00 \\
\hline $\begin{array}{l}\text { Shutdown } \\
\text { sequence, MCU } \\
\text { running on } 16 \mathrm{Mhz} \\
\text { clock }\end{array}$ & $7 \mathrm{~mA}$ & $0.62 \mathrm{~ms}$ & 4.34 \\
\hline
\end{tabular}

Fig.3. Observed current values and duration for different intervals in an RFD 


\section{$\underline{\text { Performance Analysis of a FFD }}$}

A fully functional device in a clustered home area network is either the coordinator which initiates the network and acts as sink node, or the cluster head which gathers the data from various nodes to send it to sink node. In non-beacon In its normal function, a coordinator configured in beacon enabled

TABLE III

THE NETWORK PARAMETERS

\begin{tabular}{ccc}
\hline \hline Symbol & Parameter & Value \\
\hline & Beacon order & 6 \\
$S O$ & Superframe order & 0 \\
Ret & Average no. of & 3 \\
& retransmissions & \\
$h$ & Hidden node probability & $30 \%$ \\
& Uplink data transmission & \\
$U I$ & interval & $1-100$ \\
& Downlink data & \\
$D I$ & transmission interval & 100 \\
& Beacon interval & \\
$B I$ & Network scanning interval & $0.96 \mathrm{~s}$ \\
$I_{N S}$ & & $5 \mathrm{~h}$ \\
& & 11 octets \\
$L_{A}$ & ACK frame length & $260 \mathrm{otets}$ \\
$L_{B}$ & Beacon frame length & $50 \mathrm{octets}$ \\
$L_{D}$ & Data frame length & $250 \mathrm{otets}$ \\
$L_{O}$ & Data frame overhead & 4 \\
$N_{A}$ & No. of devices & 500 \\
$N_{B}$ & No. of nodes & $128 \mu \mathrm{s}$ \\
$t_{C C A}$ & CCA analysis time & $864 \mu \mathrm{s}$ \\
$t_{A W}$ & ACK wait duration & $320 \mu \mathrm{s}$ \\
$t_{B O T}$ & Back off period & $192 \mu \mathrm{s}$ \\
$t_{I F S}$ & Interframe spacing & $19.52 \mathrm{~ms}$ \\
$t_{R E S}$ & Response time for data & \\
& request & \\
& &
\end{tabular}

$$
\mathrm{h}=\text { hours, } \mathrm{s}=\text { seconds; }
$$

topology needs only to wake in the time of beacon intervals and then it goes back to sleep if it has nothing to do.

Using a $B O$ of 6 , we can calculate the $B I$ to be 0.98 seconds.

$\mathrm{BI}=$ aBaseSuperframeDuration $\times 2^{\mathrm{BO}}$ symbols

where, aBaseSuperframeDuration $=960 \mu$ s and each symbol euals to $16 \mu \mathrm{s}$. $B I$ is an approximate data response time because the data can be only exchanged immediately after the beacon.

Superframe duration $(S D)$ determines the ON time of a device, for how long it would be awake during its contention access period time. Using $S O$ of 0 , we get an approx. $S D$ of $15 \mathrm{~ms}$. The selection of $S O$ to be equal to 0 is to minimize the ON time of a device during its contention access period. $S O$ can be increased to allow the inflow of more packets, but it will be at the expense of more energy consumption.

Function of coordinator is to maintain synchronization with the cluster heads by regularly receiving beacons from them. Duty cycle of coordinator is:

$$
\begin{aligned}
& D C_{\text {coordinator }}= \\
& \frac{t_{B T}+t_{B R}}{B I}+\frac{\left(t_{A R}\right)\left(n_{A}+n_{B}+1\right) R e t}{U I \times B I \times A}+\frac{\left(t_{S T}+t_{A R}+t_{R I}+t_{A T}\right) R e t}{D I \times B I}+\frac{t_{C A P}}{B I}+ \\
& t_{N S} / N S I
\end{aligned}
$$

where,

$t_{B T}$ is the beacon transmission time and $t_{L T}$ is the time for transmission of long data frames if any

$t_{B T}=t_{S I}+t_{I T}+L_{B} / R$

Power consumption of coordinator can be ultimately expressed as

$P_{\text {coordinator }}=\frac{E_{B T}+E_{B R}}{B I}+\frac{t_{C A P} \times P_{R X}}{B I}+\frac{\left(E_{A R}\right)\left(n_{A}+n_{B}+1\right) R e t}{U I \times B I \times A}+$

$\frac{\left(E_{S T}+E_{A R}+E_{R I}+E_{A T}\right) R e t}{D I \times B I}+\frac{t_{N S}}{N S I}+\left(1-D C_{\text {coordinator }}\right)$

(19)

where, beacon transmission energy is

$E_{B T}=t_{S I} P_{1}+\left(t_{I T}+L_{B} / R\right) P_{T X}$

(20)

The sink node/coordinator has more power as it has to initiate, transmit and received data and this power cannot be compromised. Thus it is same both for the clustered or nonclustered networks respectively.

In Figure 4, power consumption of coordinator is plotted as a function of BI. When the beacon interval goes long, power consumption of device decreases as can be observed from the graph. Same trend line (dotted line is the logarithmic trend scale) in figure 4 is observed as was for the end device but as coordinator performs most of the activities for the network and governs the network being an FFD, its power goes upto $13 \mathrm{~mW}$.
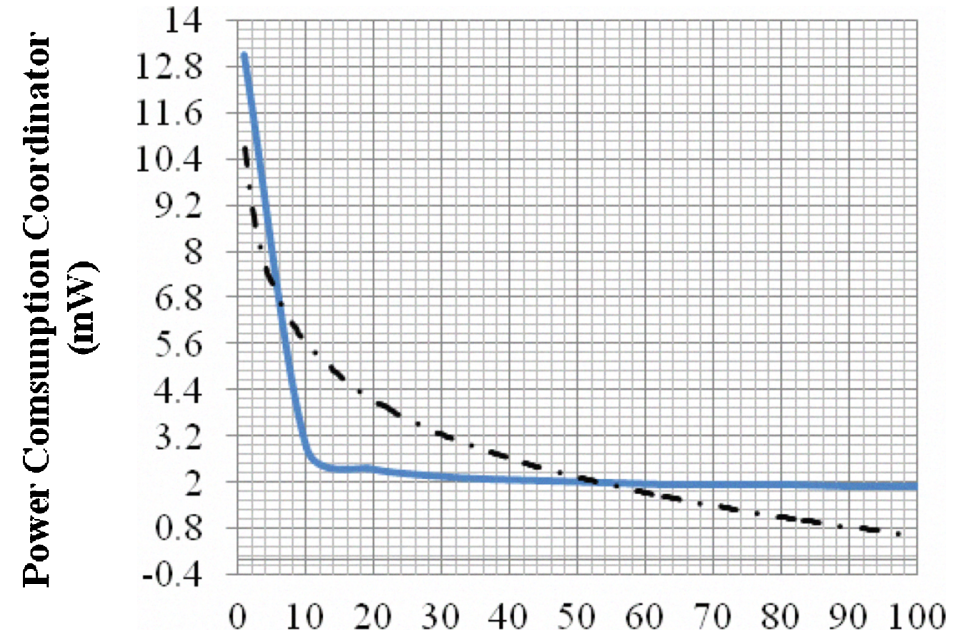

Beacon Interval (ms)

Fig. 4. Coordinator power consumption as a function of beacon interval

\begin{tabular}{|l|c|c|c|}
\hline \multicolumn{1}{|c|}{ Description } & Current & Duration & $\begin{array}{c}\text { Consumption } \\
\text { (mA.ms) }\end{array}$ \\
\hline $\begin{array}{l}\text { Startup Sequence. } \\
\text { MCU in active } \\
\text { mode running on } \\
16 \mathrm{Mhz}\end{array}$ & $5 \mathrm{~mA}$ & $0.49 \mathrm{~ms}$ & 2.45 \\
\hline $\begin{array}{l}\text { MCU ON, running } \\
\text { on 32Mhz clock }\end{array}$ & $12 \mathrm{~mA}$ & $1.8 \mathrm{~ms}$ & 21.6 \\
\hline Radio on, TX mode & $29 \mathrm{~mA}$ & $0.58 \mathrm{~ms}$ & 16.82 \\
\hline Radio on RX mode & $27 \mathrm{~mA}$ & $14.75 \mathrm{~ms}$ & 398.25 \\
\hline $\begin{array}{l}\text { Packet processing, } \\
\text { MCU ON, running } \\
\text { on 16Mhz clock }\end{array}$ & $12 \mathrm{~mA}$ & $1.1 \mathrm{~ms}$ & 13.2 \\
\hline $\begin{array}{l}\text { Shutdown } \\
\text { sequence, MCU } \\
\text { running on 16Mhz } \\
\text { clock }\end{array}$ & $7 \mathrm{~mA}$ & $0.62 \mathrm{~ms}$ & 4.34 \\
\hline
\end{tabular}

Fig.5. Observed current values and duration for 
different intervals in an FFD

Total ON time of the node $=19.34 \mathrm{~ms}$

Total active mode power consumption $=456.66(\mathrm{~mA} . \mathrm{ms})$

Total low-power mode time of node

$=1.96 \mathrm{~s}-19.34 \mathrm{~ms}=1946.66 \mathrm{~ms}$

Total current consumption in low power mode

$=500 \mu \mathrm{A} \times 1946.66 \mathrm{~ms}=0.973 \mathrm{~mA} . \mathrm{ms}$

Total active power consumption + inactive power consumption $=457.636 \mathrm{~mA} . \mathrm{ms}$

$=1.27 \times 10^{-4} \mathrm{mAh} / \mathrm{BI}=0.23 \mathrm{~mA}$

Assuming battery of 3000-mAh true capacity we have, $3000 / 0.23 \mathrm{~mA}=13043$ hours approx $=1.48$ years approx

\section{CONCLUSION}

The network formed in a cluster is well-defined consisting of leaf nodes, data aggregating and sinking heads, and the main sinking node i.e. coordinator. This structure simplifies multi hop routing and permits effective energy saving; each node has to maintain synchronization with its cluster-head only. The rest of the time, nodes may save energy by switching into sleep modes. Resulting into less energy consumption per node and hence increases network's life-time. The performance analysis shows that $B O$ and $S O$ have a very significant effect on cluster tree network and its performance. Energy consumption analysis show that using a Zigbee node in cluster tree networks like home area network increases the battery life time, by consuming less energy and hence helps in increasing life time of network.

\section{REFERENCES}

[1] Andrew Wheeler, Ember Corporation, "Commercial Applications of Wireless Sensor Networks Using Zigbee", IEEE Communication Magzine, Topics in Ad Hoc and Sensor Networks, pp 70-77, April 2007.

[2] N Liang, P Chen, T Sun, G Yang, L Chen and M Gerla, "Impact of node heterogeneity in ZigBee Mesh Network Routing", IEEE SMCC, 8-11 Oct, 2006.

[3] Edgar H. Callway, Jr,"Wireless Sensor Networks; Architectures and Protocols", pg 4-6, ISBN 0-8493-1823-8.

[4] Saad A. Khan, Hajra Aziz, Samia Maqsood, Saleha Faisal, "Clustered Home Area Network: A Beacon Enabled IEEE 802.15.4 Approach”, IEEE ICET 2008, pg 193-198, Oct 19-18 2008

[5] ZigBee Alliance. "ZigBee Specifications", version 1.0, April

2005.

[6] Bougard, B., Catthoor, F., Daly, D.C., Chandrakasan, A., Dehaene, W., "Energy efficiency if IEEE 802.15.4 standard in dense wireless microsensor networks: modeling and improvement perspectives", in Proc of Design, automation and test in Europe (DATE'05) (Munich, Germany, Maarch 7-11, 2005), IEEE, 2005, Vol. 1, 196-201

[7] G. Lu, B. Krishnamachari, and C. S. Raghavendra, "Performance evaluation of the IEEE 802.15.4MAC for low-rate lowpower wireless networks," in Proceedings of the 23rd IEEE International Performance Computing and Communications Conference (IPCCC '04), pp. 701-706, Phoenix, Ariz, USA, April 2004.
[8] Timmons, N.F.; Scanlon, W.G., "Analysis of the performance of IEEE 802.15.4 for medical sensor body area networking," Sensor and Ad Hoc Communications and Networks, 2004. IEEE SECON 2004. 2004 First Annual IEEE Communications Society Conference on, vol., no., pp. 16-24, 4-7 Oct. 2004

[9] Kohvakka, M., Kuorilehto, M., Hännikäinen, M., and Hämäläinen, T. D. 2006. Performance analysis of IEEE 802.15.4 and Zigbee for large-scale wireless sensor network applications. In Proceedings of the 3rd ACM international Workshop on Performance Evaluation of Wireless Ad Hoc, Sensor and Ubiquitous Networks (Terromolinos, Spain, October 06 - 06, 2006). PE-WASUN '06. ACM, New York, NY, 48-57

[10] www.ti.com/sc/device/CC2431

[11] J. Ha,W. H. Kwon, J. J. Kim, Y. H. Kim, and Y. H. Shin, "Feasibility analysis and implementation of the IEEE 802.15.4 multihop beacon enabled network," in Proceedings of the 15th Joint Conference on Communications \& Information (JCCI '05), pp. 1-5, Korea, April 2005.

[12] Misic J. Shafi S. Misic V.B. "The impact of MAC parameters on the performance of 802.15.4 PAN" (2005) Ad Hoc Networks, 3 (5), pp. 509-528

[13] J. Misic, S. Shafi, and V. B. Misic. "Performance of beacon enabled IEEE 802.15.4 cluster with downlink and uplink traffic", IEEE Transactions on Parallel and Distributed Systems. vol. 17. no. 4, pp. 361-376, April 2006.

[14] L. Kleinrock and F. A. Tobagi, "Packet switching in radio channels: part I-carrier sense multiple-access modes and their throughput-delay characteristics," IEEE Transactions on Communications, vol. 23, no. 12, pp. 1400-1416, December 1975.

[15] A. S. Tanenbaum, Computer Networks, Prentice-Hall, Upper Saddle River, NJ, USA, 4th edition, 2003.

[16] J. Zheng and M. J. Lee, "Will IEEE 802.15.4 make ubiquitous networking a reality?: a discussion on a potential low power, low bit rate standard," IEEE Communications Magazine, vol. 42, no. 6, pp. 140-146, June 2004.

[17] Misic, J. and Udayshankar, R. 2008. Analysis of Cluster Interconnection Schemes in 802.15.4 Beacon Enabled Networks. Int. J. Distrib. Sen. Netw. 4, 2 (Apr. 2008), 155-175

[18] Misic, J. 2007. Design and performance issues of slave-slave cluster interconnection in 802.15 .4 beacon enabled networks. Int. $J$. Parallel Emerg. Distrib. Syst. 22, 1 (Jan. 2007)

[19] J. Miši'c and C. J. Fung, "The impact of master-slave bridge access mode on the performance of multi-cluster 802.15.4 network," Computer Networks, vol. 51, pp. 2411-2449, 2007.

[20] Pollin, S. Ergen, M. Ergen, S.C. Bougard, B. Catthoor, F. Bahai, A. Varaiya, P. "Performance Analysis of Slotted Carrier Sense IEEE 802.15.4 Acknowledged Uplink Transmissions", Wireless Communications and Networking Conference, 2008. WCNC 2008 pp 1559-1564 